\title{
Generating tight-binding Hamiltonians with finite-difference methods
}

\author{
J. M. Thijssen and J. E. Inglesfield \\ University of Nijmegen, Toernooiveld, 6525 ED Nijmegen, The Netherlands
}

(Received 2 February 1995)

\begin{abstract}
A method is presented for deriving a nearest-neighbor tight-binding Hamiltonian for electrons in solids, starting from a finite-difference Hamiltonian with atomic spheres embedded in it. The space is divided into cells surrounding the atoms. The basis states of the tight-binding Hamiltonian are the eigenstates of the finite-difference Hamiltonian in these cells with zero derivative boundary conditions at the cell boundaries. To calculate the matrix elements of the full Hamiltonian, the couplings over the links crossing the cell boundaries need to be restored which leads to a coupling between states in neighboring cells. The resulting tight-binding Hamiltonian is energy independent. Typically about 100 states per cell are needed to achieve reasonable accuracy.
\end{abstract}

\section{INTRODUCTION}

Order $(N)$ methods for solving the electronic structure of solids are a sine qua non for treating systems with large unit cells, such as random systems, grain boundaries, and the Scanning tunneling microscopy tip and surface. $O(N)$ methods can be arrived at in several ways. First, there exist methods in which basis functions having a finite range in real space are constructed so that the Hamiltonian couples a restricted number of shells of neighboring atoms only. ${ }^{1-6}$ One can also exploit the diagonal form of the kinetic-energy term occurring in the Hamiltonian in Fourier space, to arrive, via fast Fourier transforming back and forth, at an $O(N \ln N)$ method (which thus scales essentially as $N) .{ }^{7}$ Recently, finite-difference methods have also received attention as a tool for solving large systems. ${ }^{8-10}$ In these methods, a finite-difference grid is defined with a grid constant much less than the linear atomic cell size, and the discretized Hamiltonian assumes a tight-binding form between grid points, the latter being obviously much more numerous than the atoms. The representation on a grid of the rapidly oscillating part of the electronic wave function close to the nuclei is poor unless the grid is very fine. One way of getting around this problem is by using pseudopotentials, ${ }^{7,10}$ which work well for many, but not all, elements.

We have recently developed a method ${ }^{11}$ that solves this problem by integrating the continuum Hamiltonian inside an atomic sphere surrounding the nucleus by standard numerical methods, and matching the solutions onto the wave function on the finite-difference grid, the latter being defined in the interstitial region only. The interior of the atomic sphere is eliminated at the price of getting an energy-dependent Hamiltonian coupling the grid points just outside the sphere. The resulting method still scales as $O(N)$ and is suitable for all elements. As the radius of the sphere can be chosen considerably smaller than the muffin tins used in methods like linear augmented plane wave and Korringa-Kohn-Rostoker (KKR), the spherical approximation for the potential inside the atomic sphere is very accurate. In this paper, we present a method for generating, starting from a finitedifference Hamiltonian (with or without an embedded atomic sphere), an energy-independent tight-binding formulation that couples states of nearest-neighbor atoms.

\section{THE METHOD}

First, the system under consideration is partitioned into cells, each cell containing a convenient number of atoms. For a periodic solid, the cells may be the parallelepiped unit cells spanned by the three unit vectors, or the Wigner-Seitz unit cell. More generally, for a nonperiodic system, the latter type of cells are Voronoi polyhedra, regions of space bounded by bisecting planes between neighboring nuclei. ${ }^{12}$ The starting point of our method is the finite-difference formulation of the Hamiltonian inside such a cell (eventually containing one or more atomic spheres) and with zero-derivative boundary conditions (ZDBC) on the cell boundaries. For points inside the cell having no neighbors outside, the Hamiltonian reads

$$
(H \psi)_{p}=\sum_{q} \frac{-1}{2 h^{2}} \psi_{q}+\left(\frac{6}{2 h^{2}}+V_{p}\right) \psi_{p}
$$

where $q$ labels the six neighbors of $p$.

The ZDBC boundary condition is realized by forcing the states $\psi$ on the grid to assume the same value at each of two points connected by a link crossing the cell boundary. So, if $p$ labels a point inside a cell having $n$ nearest-neighbor grid points outside the cell, the Hamiltonian acting in $p$ on a state $\psi$ in the cell reads

$$
(H \psi)_{p}=\sum_{q} \frac{-1}{2 h^{2}} \psi_{q}+\left(\frac{6-n}{2 h^{2}}+V_{p}\right) \psi_{p}
$$

where $q$ labels the $6-n$ neighbors of $p$ inside the cell. This boundary condition does not affect the Hermiticity of the Hamiltonian, which then has orthogonal eigenstates.

We take these states as basis states for the complete Hamiltonian, that is, the one with the couplings through the cell boundaries included. The latter obviously deviates from the ZDBC Hamiltonian on the cell boundaries only. If we denote by $(p, \alpha)$ all pairs of points connected by a link crossing the cell boundary, we can write the matrix elements of the full Hamiltonian between two ZDBC states $\psi^{(a)}$ and $\psi^{(b)}$ in the same cell (containing the point 
p) as follows:

$$
\begin{aligned}
\left\langle\psi^{(a)}|H| \psi^{(b)}\right\rangle & =E_{\mathrm{ZDBC}}^{(a)} \delta_{a b}+\frac{1}{2 h^{2}} \sum_{(p, \alpha)} \psi_{p}^{(a)} \psi_{p}^{(b)} \\
& =E_{\mathrm{ZDBC}}^{(a)} \delta_{a b}+n \psi_{p}^{(a)} \psi_{p}^{(b)}
\end{aligned}
$$

If $\psi^{(b)}$ is defined in the cell containing $\alpha$,

$$
\left\langle\psi^{(a)}|H| \psi^{(b)}\right\rangle=-\frac{1}{2 h^{2}} \sum_{(p, \alpha)} \psi_{p}^{(a)} \psi_{\alpha}^{(b)} .
$$

For a single cell with Bloch boundary conditions, we find for Bloch vector $\mathbf{k}$ :

$$
\begin{aligned}
\left\langle\psi^{(a)}|H| \psi^{(b)}\right\rangle= & E_{\mathrm{ZDBC}}^{(a)} \delta_{a b} \\
& +\frac{1}{2 h^{2}} \sum_{(p, \alpha)} \psi_{p}^{(a)}\left(\psi_{p}^{(b)}-\psi_{q}^{(b)} e^{i \mathbf{k} \cdot \mathbf{R}}\right),
\end{aligned}
$$

where $q$ is a point that transforms into $\alpha$ after a translation over $\mathbf{R}$, an integer linear combination of basis vectors of the Bravais lattice. So, with respect to the ZDBC states, the Hamiltonian assumes a tight-binding (TB) form with couplings between nearest-neighbor cells (atoms) only.

The method is somewhat less straightforward for the case when the atomic core is replaced by the embedding potential, since the normalization inside the core is unknown in that case. In order to explain this, we will first review the embedded atomic sphere method briefly here-for details, see Ref. 11. Inside the atomic spheres, we treat the Hamiltonian as a continuum operator, which can be solved rapidly and with high precision, using standard integration procedures. For a predefined energy $E$, we determine the solutions $\chi_{L}$ to the radial Schrödinger equation $[L$ stands for the combined radial quantum numbers $(l, m)]$. Inside the sphere, any solution to the Schrödinger equation can be written as $\sum_{L} a_{L} \chi_{L}$. The finite-difference grid is defined in the interstitial region. A state $\psi$, which is defined on this grid, determines the expansion coefficients $a_{L}$ of the solution inside the atomic sphere uniquely, if one assumes the radial solution to be valid up to the grid points just outside the sphere. Defining an inner product on the sphere boundary,

$$
\langle\psi \mid \phi\rangle_{B}=\sum_{(p, \alpha)} \psi_{p} \phi_{\alpha}
$$

where $(p, \alpha)$ are the links crossing the sphere boundary, $p$ lying just outside, $\alpha$ just inside the sphere, one finds an expression for the expansion coefficients $a_{L}$ of the solution inside:

$$
a_{L}=\sum_{L^{\prime}} S_{L L^{\prime}}^{-1}\left\langle\psi \mid \chi^{L^{\prime}}\right\rangle_{B}
$$

where the inverse of the overlap matrix $S^{L, L^{\prime}}=$ $\left\langle\chi^{L} \mid \chi^{L^{\prime}}\right\rangle_{B}$ occurs. Knowing the $a_{L}$, the action of the finite-difference Hamiltonian can be calculated on all the points on the finite-difference grid, even those lying near the sphere boundary and coupling to points inside this sphere. The procedure outlined here can formally be presented as coupling all the points just outside the atomic sphere. However, using such a coupling is computationally redundant: first calculating the coefficients $a_{L}$ and then using them to calculate the contributions to the Hamiltonian acting on the points $p$ is an order $N$ procedure.

Diagonalizing the grid Hamiltonian, we get a spectrum with an eigenvalue which, at self-consistency, should match the energy at which the $\chi^{L}$ were calculated. The energy-derivative of the Hamiltonian can easily be calculated via the energy derivative of the $\chi_{L}$. This energy derivative of the Hamiltionian is used to accelerate the self-consistency iteration procedure for the energy.

The resulting eigenstates as they come out of the diagonalization procedure are normalized on the finitedifference grid. Of course, eigenstates at different energies need not be orthogonal. However, they are calculated using a Hamiltonian which represents the full, energy-independent Hermitian Hamiltonian which has orthonormal eigenstates. We call the latter $\mathcal{H}$, and its eigenstates and eigenvalues $\Psi_{i}$ and $E_{i}$. The Hamiltonian in the interstitial region with the energy-dependent boundary condition is called $H-V(E)$. Its eigenvalues are of course equal to those of $\mathcal{H}$ and its eigenstate $\phi_{i}$ at energy $E_{i}$ is proportional to $\Psi_{i}$ in the interstitial region with proportionality constant $\alpha_{i}$ :

$$
\phi_{i}\left(\mathbf{r}_{I}\right)=\alpha_{i} \Psi_{i}\left(\mathbf{r}_{I}\right)
$$

for the points $\left(\mathbf{r}_{I}\right)$ of the interstitial grid.

Since both Hamiltonians have the same Green function in the interstitial region, ${ }^{11}$ the matrix elements $\left\langle\phi_{i}|H-V(E)-E| \phi_{i}\right\rangle$ and $\left\langle\Psi_{i}|\mathcal{H}-E| \Psi_{i}\right\rangle$ must be equal. Expanding $V(E)$ to first order around $E_{i}$, one finds that

$$
\alpha^{2}=\left[1-\left\langle\phi_{i}\left|\frac{d V\left(E_{i}\right)}{d E}\right| \phi_{i}\right\rangle\right]^{-1},
$$

so that the $\phi_{i}$ can be normalized and thus form an orthonormal set with respect to the full inner product (including the interiors of the atomic spheres).

\section{RESULTS}

We have implemented the method and tested it for fcc and simple cubic copper, using a muffin-tin potential generated elsewhere. Using the finite-difference program with an embedded atomic sphere inside, we have determined the eigenstates of the ZDBC Hamiltonian. We generated sets containing up to 113 of such states. Such a set was then used as input for the tight-binding program. The calculation of the matrix elements of the Hamiltonian for two ZDBC states is given in Eq. (5) and is particularly simple since the Bloch phase factor $e^{i \mathbf{k} \cdot \mathbf{R}}$ is common to all pairs of points connected through one face of the cell. The time consuming step, therefore, is the determination of the ZDBC states, after which the bands can be generated very fast.

The results for both lattices are comparable, so we restrict the presentation to the fcc structure. Figure 1 shows the band structure on the $\Gamma-X$ segment of the Brillouin zone, using various numbers of $\mathrm{ZDBC}$ states in the cell, using a grid constant $h=a / 18$, where $a$ is the fcc lattice constant. It is observed that the band structure converges with increasing numbers of states to the exact 


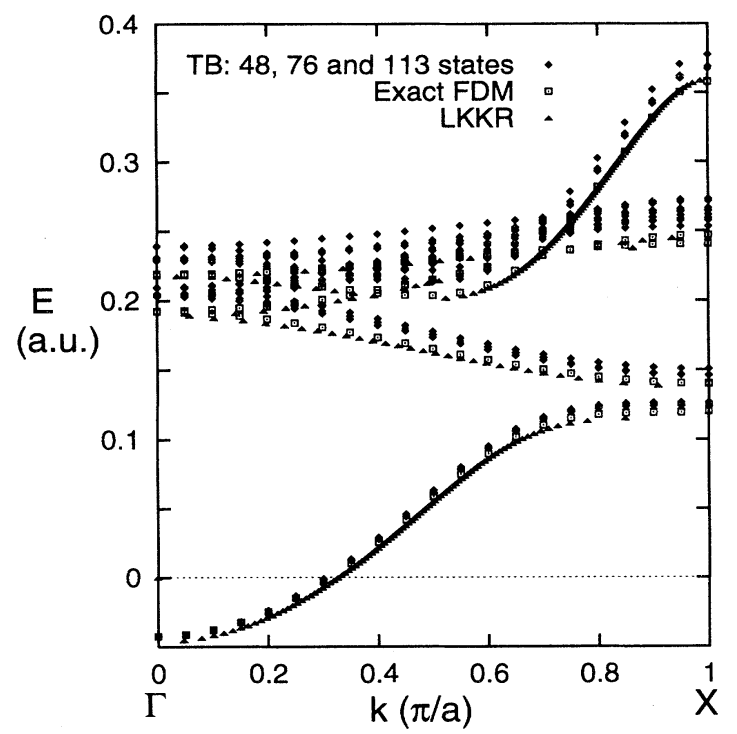

FIG. 1. Energy bands for fcc copper for various numbers of TB states. The fcc lattice constant $a$ is 6.8219 a.u. and the finite-difference grid constant $h$ is taken to be $a / 18$. The nucleus is placed at the center of the Wigner-Seitz unit cell. The embedding sphere has a radius of 1.4 a.u. All bands determined with the TB method described in this paper are represented by diamonds. The highest of these correspond to a basis consisting of 48 states, the lowest to a basis of 113 states. The squares represent the "exact" finite-difference band structure, thus without using the tight-binding method. The essentially exact LKKR band structure is shown with triangles.

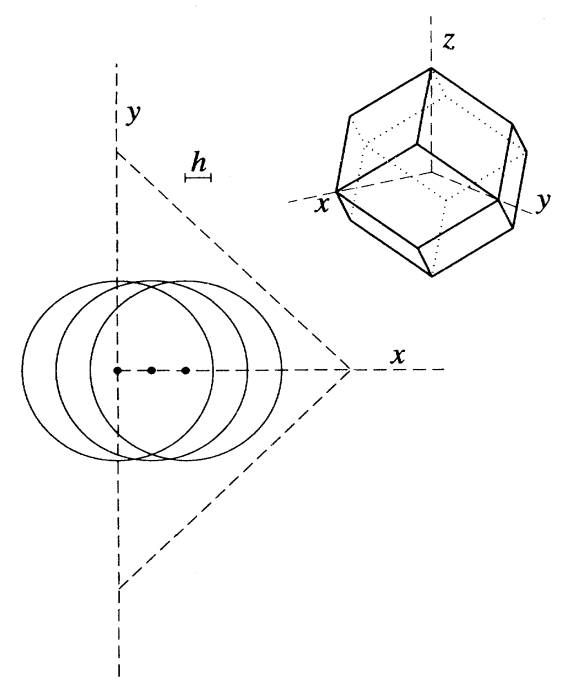

FIG. 2. Different positions of the embedding sphere in the Wigner-Seitz unit cell, which is shown in the upper right corner. The atomic nucleus lies at the center of the embedding sphere in all cases. The finite-difference grid constant $h$ is represented. Displacements of the sphere from the origin are $0,0.5$, and 1.0 a.u., respectively.

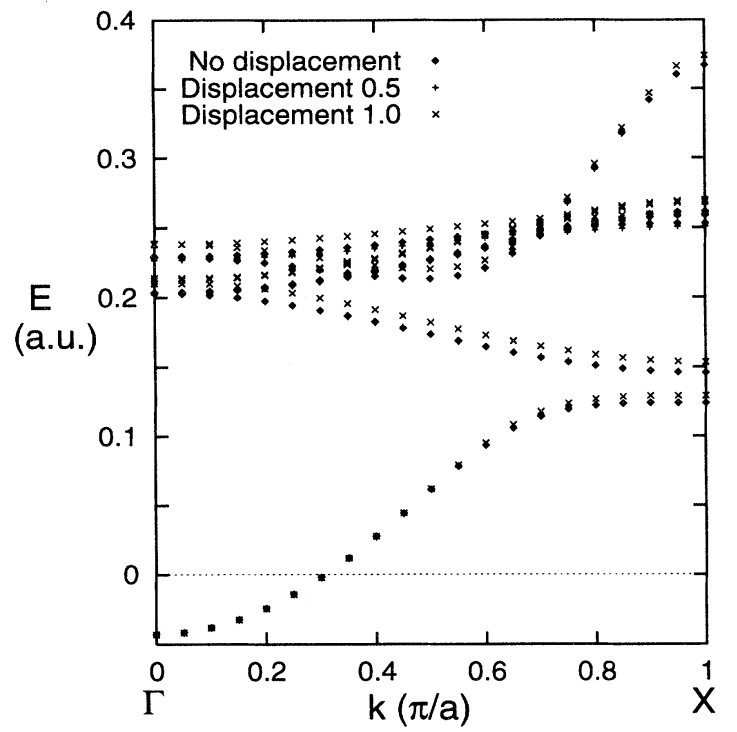

FIG. 3. Variation of the band structure for the different positions in the Wigner-Seitz cell shown in Fig. 2 .

finite-difference result, which is also represented in Fig. 1. For 113 states, the accuracy of the TB bands is better than 0.01 a.u., which is sufficient for most applications. The essentially exact layer-KKR band structure is also shown in Fig. 1.

Of course, in a system without periodicity, the spheres will not be located right at the center of a regular polyhedron. To study the effect of a nonsymmetric environment, we have calculated the band structure in the $\Gamma-X$ segment for three different positions of the sphere along the $X$ axis (see Fig. 2). The band structures are shown in Fig. 3. It is seen that shifting the sphere a little does not degrade convergence noticeably. However, if the sphere is shifted so far that it is about as close to the cell boundary as the grid constant of the finite-difference grid, one needs significantly more than $\sim 100$ states to achieve satisfactory results.

\section{DISCUSSION}

The tight-binding method presented here provides a way of calculating band structures with an energyindependent, strictly nearest-neighbor, tight-binding Hamiltonian. One can imagine using boundary conditions other than a zero derivative on the cell boundary. This may eventually increase the convergence so that fewer states are needed. ${ }^{13}$

In the case of a periodic solid, like the copper example presented above, the method is very efficient since the ZDBC set of states has to be calculated only once and can then be used for every $k$ point. For a large system, the number of $k$ points needed is inversely proportional to the number of atoms. Therefore, one uses only one type of boundary condition for very large systems. If we have to determine the ZDBC states for every Voronoi cell, the only time gain is to be expected from the fact 
that the resulting tight-binding Hamiltonian is energy independent. However, since we need many ZDBC states, the tight-binding method will probably be slower than the original finite-difference method of Ref. 11. The materials for which the method might be efficient are for example random alloys and quasicrystals. In the first example, one can take two different types of atoms, randomly distributed over, say, an fcc lattice. In that case one has to find two sets of ZDBC states only, which can then be used in the whole system. The example treated here shows that the ZDBC wave functions can be used to find the actual wave function even though this satisfies more complicated boundary conditions at the surface of the cell. Hence the efficiency of the method for heterogeneous systems is expected to comparable with the case presented here. For quasicrystals, a similar approach can be followed.

Comparing with other methods using finite-range Hamiltonians, one must realize that the method presented here has the advantage of being essentially fullpotential, since the radius of the atomic sphere is taken much smaller (1.4 a.u.) than that for touching or over- lapping spheres. In the tight-binding LMTO method by Andersen and Jepsen, ${ }^{3-5}$ the structure constants defining the range over which atomic states are coupled, are cut off typically beyond second nearest neighbors. This method is, however, very economical in the sense that a minimal amount of states is taken into account: for every $L=(l, m)$ just once. For a fcc structure, this leads to an Hamiltonian matrix having $18 \times 9$ nonzero entries per atomic site, to be compared with the $100 \times 12$ nonzero entries in our method. The latter, however, takes all spherical harmonics up to and including $l=4$. For the (more accurate) tight-binding form of the KKR method, ${ }^{6}$ such short range cannot be achieved for the structure constants. Moreover, the Hamiltonian is energy dependent in this method.

\section{ACKNOWLEDGMENTS}

Support from the Human Capital and Mobility Network " $A b$ initio (from electronic structure) calculation of complex processes in materials" (Contract No. ERBCHRXCT930369) is acknowledged.
${ }^{1}$ D.G. Pettifor, Phys. Rev. Lett. 63, 2480 (1989).

${ }^{2}$ M. Aoki, Phys. Rev. Lett. 71, 3842 (1993).

${ }^{3}$ O.K. Andersen and O. Jepsen, Phys. Rev. Lett. 53, 2571 (1984).

${ }^{4}$ O.K. Andersen, O. Jepsen, and D. Glötzel, in Highlights of Condensed-Matter Theory, edited by F. Bassani, F. Fumi, and M.P. Tosi (North-Holland, New York, 1985).

${ }^{5}$ O.K. Andersen, Z. Pawlowska, and O. Jepsen, Phys. Rev. B 34, 5253 (1986).

${ }^{6}$ L. Szunyogh, B. Ujfalussy, P. Weinberger, and J. Kollár, Phys. Rev. B 49, 2721 (1994).

${ }^{7}$ L.-W. Wang and A. Zunger, J. Phys. Chem. 98, 2158
(1994).

${ }^{8}$ S. Baroni and P. Gianozzi, Europhys. Lett. 17, 547 (1992).

9 J.B. Pendry, A. Prêtre, P.J. Rous, and L. Martín-Moreno, Surf. Sci. 244, 160 (1991).

10 J.R. Chelikowsky, N. Troullier, and Y. Saad, Phys. Rev. Lett. 72, 1240 (1994).

11 J.M. Thijssen and J.E. Inglesfield, Europhys. Lett. 27, 65 (1994).

12 G. Voronoi Reine, Angew. Math. 134, 198 (1908).

${ }^{13}$ P.G. Burke and K.A. Berrington, in Atomic and Molecular Processes, an R-Matrix Approach, edited by P.G. Burke and K.A. Berrington (Institute of Physics, Bristol, 1993). 